\title{
Problèmes théoriques et pratiques liés à l'élevage et à la production de masse des Culicides
}

\author{
par M. COLUZZI \\ Institut de Parasitologie, Université de Rome \\ Rome, Italie
}

\begin{abstract}
Résumé
Les problèmes théoriques et pratiques de l'élevage des Culicides sont analysés, particulièrement en vue de leur production de masse dans le cadre de programmes de lutte génétique.

L'élevage des moustiques a fait, ces dernières années, des progrès considérables grâce surtout aux recherches sur les insecticides. Pour plusieurs des espèces d'intérêt sanitaire on a pu définir un milieu de culture suffisamment simple et reproductible. Toutefois les exigences alimentaires des larves devraient être mieux précisées ainsi que le rôle joué dans leur développement par les micro-organismes associés aux aliments.

Les obstacles les plus importants ont été signalés au niveau du comportement de l'adulte dont les cycles d'activité sont quelquefois très difficiles à obtenir en cage. Ces difficultés peuvent être souvent surmontées par une étude des interactions entre milieu artificiel et rythmes endogènes. Néanmoins chez de nombreuses espèces l'accouplement, le repas de sang ou la ponte doivent être plus ou moins forcés par l'utilisation de techniques spéciales qui s'appliguent mal à la production à grande échelle.

En considérant l'utilisation des moustiques de laboratoire dans des programmes de lutte génétique, leur compétitivité a une importance fondamentale et demande une évaluation aussi précise que possible. Le manque de compétitivité peut être dû à un abaissement de vigueur conséquent à une adaptation forcée au laboratoire ou bien il peut être lié aux changements génétiques qui ont lieu dans les souches entretenues depuis plusieurs générations au laboratoire. Ces changements génétiques devraient être étudiés, surtout par rapport à leur influence sur la mobilité et sur le comportement sexuel de l'insecte.
\end{abstract}




\section{Summary}

The theoretical and practical problems of laboratory culture of mosquitoes are discussed with particular reference to mass production for genetic control.

Over the last few years there have been great advances in mosquito breeding techniques thanks to the research associated with insecticide resistance. Fairly simple and reproducible culture media are available for most species of medical importance. However, further information is needed on food requirements of the larvae and on the rôle of micro-organisms in larval development.

The most important obstacles in mosquito-rearing involve adult behaviour, as the activity cycles are sometimes very difficult to reproduce in the cage. Such difficulties may often be overcome by studying the interactions between the artificial environment and the endogenous rhythms. However in the case of many species, mating, blood feeding and oviposition need to be more or less induced by special techniques, wich represent a clear limit to mass production.

In view of the utilization of mosquitoes reared in laboratory for genetic control, the competitivity of the individuals released is of fundamental importance and must be evaluated as precisely as possible. The inability to compete with the natural population may depend on low vigour resulting from forced adaptation to the laboratory. It may also reflect genetic changes that are expected to occur after many generations of laboratory rearing. These genetic changes should be studied mainly with respect to their importance on mosquito mobility and sexual behaviour.

Les méthodes de lutte génétique expérimentées récemment contre les vecteurs se basent, comme on l'a exposé dans d'autres communications de ce Colloque, sur l'introduction, dans les populations naturelles, d'individus élevés en milieu artificiel et destinés à enrayer la reproduction de l'espèce à combattre. L'élevage de l'espèce au laboratoire, qui représente une étape importante pour le progrès de toute connaissance sur la biologie de l'organisme colonisé, est évidemment une condition essentielle en matière de lutte génétique. Parmi les arthropodes hématophages, cette méthode de lutte est envisagée surtout contre les moustiques, et c'est aux techniques d'élevage de ces insectes (des espèces méditerranéennes en particulier), que se rapporte notre exposé.

L'élevage des moustiques a fait, ces dernières années, des progrès considérables grâce surtout aux recherches sur les insecticides. Les principales techniques d'élevage ont été résumées lors d'un Symposium organisé en 1963, à Gainesville (Floride), les comptes rendus de ce colloque ont été publiés dans le Bulletin de l'Organisation Mon- 
diale de la Santé (vol. 31, 1964, pp. 433-622). D’autres importantes contributions sont incluses dans le livre édité par Smith concernant l'élevage des insectes et leur production de masse (Insect colonization and mass production, Academic Press, 1966). La publication de Trembley (1955) reste fondamentale pour toute référence antérieure à $1954\left({ }^{*}\right)$.

Le but de cette communication n'est pas de décrire les différentes techniques mises au point, mais plutôt, d'analyser quelques-uns des facteurs qui, sur la base de l'expérience acquise, peuvent influencer le succès d'un élevage.

\section{Le développement en milieu artificiel.}

Les succès d'un élevage dépendent, avant tout, de la possibilité d'obtenir au laboratoire des conditions favorables au développement de l'espèce à élever. Les obstacles peuvent être à la fois théoriques et pratiques: provenir soit du manque de connaissances sur l'écologie et la physiologie de l'espèce, soit de difficultés techniques permettant la réalisation en laboratoire, de biocénoses complexes. Le milieu des larves rhéophiles des simulidés est, par exemple, caractérisé par des facteurs tels que le mouvement et l'oxygénation de l'eau, la disponibilité continuelle de nourriture, dont la manipulation pose plusieurs problèmes encore irrésolus (voir Muirhead-Thomson, 1966). De même, les conditions des gîtes larvaires de quelques espèces de moustiques sont très difficile à réaliser artificiellement: pour s'en tenir aux Culicides méditerranéens, nous avons rencontré des obstacles considérables avec Anopheles marteri, dont les larves colonisent les ruisseaux de montagne. Heureusement, cela n'est pas le cas de la plupart des moustiques d'intérêt sanitaire, dont le développement est obtenu assez facilement au laboratoire. Le problème reste, toutefois, de définir un milieu de culture standard et reproductible, ce qui revient à dégager et à contrôler les principaux facteurs qui influencent le développement et la vitalité de l'espèce en introduisant, dans le milieu artificiel, le nombre minimum de variables.

La mise au point d'un milieu de culture pour une espèce donnée de moustique devrait partir de certaines connaissances de base sur les besoins alimentaires des larves. En effet, la complexité du phyto- et du zooplancton des gîtes larvaires, et la présence de différents micro-organismes dans le régime naturel des Culicides (voir Laird, 1956) rendent difficile l'étude expérimentale des substances nutritives essentielles, et de leur origine. Des élevages en conditions aseptiques ont été réalisés avec Aedes aegypti, et des régimes chimiquement définis ont été proposés au moins pour cette espèce (cf. Akov, 1962) toutefois, en pratique, l'établissement et l'entretien des colonies de moustiques a procédé plutôt de l'empirisme par tentatives successives.

Les premiers essais d'élevage des larves d'Anopheles (voir Trambley, 1955 pour les références) se basaient sur l'utilisation de la boue, de la végétation, et quelque fois de l'eau même du gîte larvaire, avec le danger d'introduire dans l'insectarium, des organismes pathogènes ou même des prédateurs, et de rendre impossible toute stan-

(*) Un nouveau "Manual for mosquito rearing and experimental techniques» a été publié par E. J. Gerberg en 1970. (Bulletin $\mathbf{N}^{\circ} 5$ of the American Mosquito Control Association). 
dardisation. Dans le but de simplifier ces milieux de culture semi-naturels, on a expérimenté de très nombreux régimes. Une liste des différentes formulations utilisées comprend pratiquement tous les groupes principaux des composés organiques d'origine végétale ou animale autre que levures, algues et différents types de micro-organismes (voir Asahina, 1964). Actuellement, les larves de nombreuses espèces sont élevées dans l'eau distillée ou de fontaine (dont certains caractères physico-chimiques sont éventuellement modifiés), et pour leur alimentation, on utilise des produits pour animaux de laboratoire ou pour enfants, ajoutés à l'eau sous forme de poudre non mouillable (dans le cas des Anopheles) ou de suspension (dans le cas des Culicinés). Ce type d'aliment s'est montré satisfaisant pour l'entretien de presque toutes les espèces, mais le milieu de culture obtenu pose souvent des problèmes de pollution par bactéries, champignons et protozoaires. D'ailleurs, les facteurs stimulants le développement des larves sont liés à certains de ces micro-organismes. Hazard et al. (1967) ont fait la revue de cet important aspect, et ont confirmé un effet favorisant des infusions de foin sur le développement de cinq espèces de moustiques; ils pensent que la source des substances stimulantes sont les bacteria ou les produits de leur métabolisme. Certains protozoaires ou algues pourraient également favoriser le développement d'autres espèces (voir Asahina, 1964).

La végétation aquatique, en tant que facteur d'oxygénation, peut être facilement remplacée par une aération mécanique, de sorte que des plantes submergées peuvent être complètement éliminées du gîte artificiel. Même dans le cas des larves de Mansonia, il est possible de remplacer les plantes support par un papier imperméable maintenu à la surface de l'eau (Laurence et Smith, 1958).

La durée du cycle de développement a une importance primordiale pour l'élevage et ce sont généralement les espèces homodynames, à cycle de développement rapide, qui s'adaptent le mieux en laboratoire. La longueur du cycle larvaire majore, en effet, les difficultés de stabilisation du milieu qui souvent évolue d'une manière imprévisib'e. D'ailleurs, un cycle long traduit souvent une adaptation à un milieu naturel soumis à une dynamique complexe, difficilement reproductible au laboratoire. Ainsi, la constance des principales variables physiques (températures, humidité relative et lumière) qui caractérisent le milieu artificiel peut avoir un effet dramatique sur des espèces dont le cycle annuel comporte des périodes de diapause obligatoire.

Les phénomènes de quiescence et de diapause peuvent être à l'origine de difficultés considérables (cf. Clément, 1963). Ils devraient faire l'objet d'études précises. Le rôle très important de la photopériode dans la biologie des espèces polycycliques hétérodynames est souvent sous-évalué. Une photopériode longue, réglée par rapport à la période de l'année où l'évolution est la plus rapide, s'impose pour l'élevage des nombreuses espèces de moustiques des régions tempérées. Cette variable devrait être également prise en considération dans l'élevage des espèces subtropicales (voir Danilevskii, 1965). Des photopériodes mal réglées pourraient être à l'origine des variations périodiques de fécondité et de développement observées par de nombreux auteurs chez

(*) Un régime tout à fait différent est néanmoins utilisé dans le cas des larves prédatrices de Toxorhynchites nourries avec des larves d'autres espèces de moustiques. 
des colonies de moustiques entretenues depuis longtemps au laboratoire. L'importance des phénomènes de diapause doit être également considérée au moment de la colonisation ; on peut, en effet, arriver à des échecs complets en essayant d'adapter au milieu du laboratoire un matériel qui, sur la base du photopériode expérimentée dans la nature, est «préparé » à des conditions tout à fait différentes.

\section{Le comportement en milieu artificiel.}

S'il est relativement facile, en disposant de moyens techniques, d'établir des conditions climatiques assurant la vitalité de l'insecte adulte, il peut être, au contraire, problématique d'obtenir en captivité les variations journalières d'activité qui caractérisent la biologie de l'espèce. A côté d'espèces qui, grâce à leur comportement plastique, sont facilement adaptables aux conditions du laboratoire (remarquons qu'il s'agit souvent d'éléments sténotopes et domestiques), il y en a d'autres sur lesquelles le milieu artificiel produit des réactions aberrantes ou des états d'inactivité presque complète très difficile à renverser. Un examen de la bibliographie sur l'élevage des moustiques montre que c'est au niveau du comportement des adultes qu'on rencontre les obstacles les plus importants.

La copulation est en rapport avec le vol nuptial chez la plupart des espèces de moustiques et il est évident que ce comportement sexuel spécialisé et influencé négativement dans une cage de laboratoire. L'absence de copulation en cage est, en effet, une des causes les plus fréquentes d'échec. Roubaud (1932) distingue ainsi les espèces «sténogames » et «eurygames » selon que l'insémination a lieu ou non en espace limité. En réalité, cette distinction n'est pas toujours facile : la «sténogamie » représente clairement chez quelques moustiques, comme les formes domestiques de Culex pipiens, une adaptation développée secondairement par rapport à l'occupation d'un milieu particulier, tandis que, dans d'autres cas, elle est seulement la manifestation d'un comportement sexuel plus ou moins compatible avec les conditions du laboratoire. Au sein du complexe maculipennis, il n'existe probablement pas une véritable «sténogamie » comparable à celle, caractéristique, d'Aedes aegypti ou Culex pipiens fatigans, mais il n'y a aucun doute, néanmoins, que l'activité sexuelle de atroparvus se développe en espace limité plus facilement que celle de messeae ou sacharovi. D'ailleurs, la plasticité de ce comportement semble varier parmi des différentes populations géographiques de la même espèce, et aussi parmi les individus de la même population. A propos d'Anopheles quadrimaculatus, Gahan (1966) remarque qu'un des problèmes les plus difficiles est d'obtenir, au laboratoire, l'insémination des femelles de la première génération et il ajoute : « Les échecs sont fréquents et la récolte du matériel dans la nature doit être poursuivie jusqu'au moment où l'on obtient quelques specimens dont la progéniture s'accouple au laboratoire. » On admet implicitement dans cette observation comme dans d'autres similaires sur différentes espèces de moustiques, que le succès de l'élevage se base sur la sélection de caractères particuliers de comportement. Néanmoins, la contribution des facteurs du milieu à la variabilité du comportement sexuel est considérable. L'éclairement joue un rôle fondamental (voir Bates, 1941 ; Nielsen ct Nielsen, 1962); ainsi des variations significatives dans le pourcentage des femelles 
inséminées peuvent être observées dans des souches entretenues depuis longtemps au laboratoire selon que la cage est, par exemple, sous l'influence de lumière constante ou naturellement décroissante (Gahan et Smith, 1964). Une très grande importance a été donnée également aux dimensions des cages, qui dans les premières expériences d'élevage de moustiques étaient d'environ $1 / 4$ de mètre cube ou plus grandes. Actuellement, les cages les plus fréquemment utilisées sont d'environ $1 / 30^{\circ}$ de mètre cube, et de très petites cages de $9 \times 9 \times 7 \mathrm{~cm}$ ont été employées par d'Alessandro et al. (1961) pour obtenir la copulation d'Anopheles labranchiae. La hauteur de la cage devrait favoriser l'insémination des espèces dont la copulation, qui débute en vol, semble être arrêtée par le contact avec une paroi (cf. Clément, 1963). Il est toutefois probable que la densité des adultes a aussi quelque importance comme le remarquent D'Alessandro et al. (1961). D'ailleurs, il y a des espèces de moustiques (par exemple Anopheles maculipennis s.s. ou Aedes caspius) chez lesquelles l' « eurygamie » apparaît comme un caractère particulièrement rigide, et l'activité sexuelle en cage ne semble être stimulée par aucune variation du milieu. Actuellement, l'élevage de telles espèces n'est possible que grâce à la technique d'accouplement forcé décrite par McDaniel et Horsfall (1957) et diversement appliquée par de nombreux auteurs (cf. Coluzzi, 1962 ; Baker, 1964).

Des obstacles importants à l'élevage des moustiques ont été également rencontrés au niveau de l'activité trophique des adultes. Le rapport parasite-hôte plus ou moins étroit, qui caractérise l'alimentation des arthropodes hématophages, est un phénomène quelquefois très difficile à reproduire au laboratoire. Ainsi, le repas de sang constitue un problème d'importance primordiale dans l'élevage des Ixodidés (voir Gregson, 1966) et aussi dans l'élevage de quelques groupes de nématocères piqueurs (Simulidés en particulier). Chez les moustiques, on observe de très fortes différences d'une espèce à l'autre. Le repas de sang d'Aedes aegypti au laboratoire est obtenu avec facilité à n'importe quelle heure du jour, ou dans n'importe quelle condition de lumière; ce moustique peut d'ailleurs être nourri sur des vertébrés très différents, ainsi que sur des membranes artificielles. Au contraire, d'autres espèces, non moins agressives qu'Aedes aegypti dans la nature, se refusent à se nourrir dans une cage d'élevage et dans quelques cas seulement, la piqûre est stimulée par le contact direct avec la peau de l'hôte. Des différences de ce type ont été observées, par exemple, entre des espèces jumelles du complexe gambiae du genre Anopheles dont les espèces A et B piquent beaucoup plus facilement au laboratoire que l'espèce $\mathrm{C}$ (Davidson, communication personnelle). Ces échecs à obtenir le repas de sang malgré toute stimulation sont probablement dus à l'influence négative du milieu artificiel sur les rythmes endogènes qui règlent, de façon plus ou moins stricte, l'activité trophique de ces insectes (cf. Clément, 1963). Cela est confirmé par le fait qu'il est quelquefois suffisant d'offrir l'hôte à des heures différentes, pour avoir des variations significatives dans le pourcentage des femelles gorgées.

La rétention des œufs mûrs est un autre phénomène fréquemment observé dans les populations des moustiques au laboratoire. Pour surmonter cet obstacle, il est évidemment nécessaire de réaliser une étude précise, pour chaque espèce, des variables 
du milieu influençant l'oviposition. Les expériences portant sur le choix du lieu de ponte ont démontré la grande complexité de cette activité chez quelques espèces de moustiques (voir Bates, 1948). Dans certains cas, les facteurs stimulant la ponte peuvent être d'une telle complexité, qu'on est obligé d'appliquer des techniques d'oviposition forcée. La décapitation des femelles gravides a été, par exemple, utilisée avec succès par de Coursey et Webster (1959) avec Aedes sollicitans, tandis que Kreutzer et Kitzmiller (1969) ont réussi à obtenir des œufs de Anopheles earlei en obligeant les femelles, par l'amputation d'une aile, à rentrer en contact avec une surface d'eau.

\section{Les problèmes liés à la production de masse.}

Un procédé d'élevage de masse pour Aedes aegypti a été mis au point et expérimenté avec succès (Fay et Morlan, 1959, Morlan, 1966), et la même technique peut être adaptée avec peu de modifications à d'autres moustiques. Toutefois, seules quelquesunes des nombreuses espèces entretenues à présent en laboratoire montrent une vitalité et une capacité reproductive, en milieu artificiel, comparables à celles d'Aedes aegypti et pour très peu d'entre elles, il est possible d'envisager un élevage de masse aussi productif. La plasticité de comportement, la rapidité du cycle vital, l'uniformité de développement, la résistance aux manipulations et la tolérance aux densités élevées facilitent certes l'entretien des espèces au laboratoire. Ces caractères deviennent de la plus haute importance lorsqu'on doit passer de l'élevage expérimental à l'élevage de masse. Lorsqu'il s'agit de produire des dizaines de milliers d'individus, le contrôle du coût de la production s'impose, et ne peut être obtenu que par une mécanisation aussi poussée que possible des procédés utilisés. Il en découle que les procédés d'élevage tels que nous les avons présentés, dont la réussite peut être obtenue par l'application de techniques spéciales (copulation forcée) ou par le soin attentif et continu du chercheur, ne peuvent s'appliquer à la production à grande échelle.

Les problèmes les plus complexes liés à l'élevage de masse concernent la qualité des organismes produits plutôt que leur quantité : en considérant l'utilisation du matériel dans des programmes de lutte génétique, le niveau de compétitivité des individus à libérer dans la nature a une importance fondamentale, et l'évaluation des causes de variation de ce paramètre est souvent très difficile. Les expériences des lâchers de mâles stériles faites avec certaines espèces de moustiques semblent indiquer que la compétitivité de ces insectes est particulièrement influencée par les conditions d'élevage. Un manque presque total de compétitivité des mâles de laboratoire par rapport à ceux de la nature a été observé dans les expériences faites en Floride avec une vieille colonie d'Anopheles quadrimaculatus (Weidhaas et al., 1962 ; Dame et al., 1964). De bas degrés de compétitivité du matériel de laboratoire ont été ainsi observés avec Aedes aegypti (Morlan et al., 1962) et avec Culex fatigans (Patterson et al., 1968 a).

Plusieurs facteurs inhérents au milieu artificiel peuvent influencer l'expression des « caractères » physiologiques et éthologiques (longévité, capacité de dispersion, activité sexuelle) qui sont à la base de la compétitivité de l'insecte. Le manque de compétitivité peut être génériquement dû à une adaptation forcée au laboratoire dont dérive un abaissement de vigueur souvent plus ou moins masqué dans le milieu constant de 
l'insectarium, mais très évident dans la nature. En étudiant la longévité d'A. quadrimaculatus élevé au laboratoire, et entretenu dans des cages soumises aux conditions climatiques externes, Patterson et al. $(1968 \mathrm{~b})$ ont constaté que seulement $50 \%$ des mâles et $78 \%$ des femelles survivaient après $24 \mathrm{~h}$, et que moins de $1 \%$ des mâles et $4 \%$ des femelles étaient encore vivants après $72 \mathrm{~h}$, tandis que les mêmes moustiques montraient une vitalité presque normale en chambre climatisée.

Le manque de compétitivité peut être lié également aux changements génétiques qui ont lieu dans les souches entretenues depuis plusieurs générations au laboratoire. L'importance du degré d'hétérozygotie par rapport à la vigueur et au comportement sexuel en particulier, a été souligné par divers auteurs (voir Boesiger dans ce même Colloque), et on sait que les différences de comportement peuvent être rapidement sélectionnées au laboratoire. Bien que la plupart de ces données dérivent d'études sur Drosophila, et que l'on possède très peu de données sur la génétique du comportement des moustiques (Mattingly, 1967), il n'y a aucune raison de douter que des colonies de ces insectes puissent être sujets à des changements génétiques capables d'influencer leur compétitivité vis-à-vis des populations naturelles. D'ailleurs, la pression sélective à laquelle sont soumises les colonies de moustiques est souvent très importante et, comme nous l'avons remarqué précédemment, une sélection évidente a lieu au moment de la copulation en cage des espèces partiellement «sténogames » lorsqu'on observe, dans les premières générations colonisées, un pourcentage de femelles inséminées fréquemment, inférieur au $20 \%$. Une sélection de certains types de comportement sexuel (sténogamie) pourrait expliquer au moins en partie, le manque de compétitivité des mâles de $A$. quadrimaculatus utilisés dans les expériences de lutte génétique exécutées en Floride (Dame et al., 1964).

Dans les colonies de laboratoires, d'autres processus sélectifs peuvent modifier les caractères de comportement, en particulier, ceux qui conditionnent la mobilité et la capacité de dispersion de l'insecte. Les moustiques les plus mobiles semblent avoir une faible valeur adaptative dans les cages blanches de nos insectariums où ils sont très probablement remplacés par un variant moins sensible à certains stimulus. Nous avons eu effectivement l'occasion de noter, lors d'études sur l'irritabilité des Anopheles au DDT, une mobilité significativement moins élevée des individus d'une souche d'A. atroparvus entretenue depuis plus de 30 générations au laboratoire, par rapport à celle des individus ayant la même origine géographique, mais élevés en milieu artificiel pendant une seule génération. En travaillant avec la même espèce, Gérold et Laarman (1967) ont récemment démontré la possibilité d'obtenir, par sélection, de remarquables différences de mobilité.

En ce qui concerne les moustiques du midi méditerranéen, l'eurigamie de la plupart des espèces constitue la limitation principale à leur élevage de masse. A l'occasion de nombreux essais d'élevage, nous avons enregistré des pourcentages d'insémination proches de $100 \%$, à la première génération en cage, seulement avec Culex pipiens (populations domestiques), Culiseta longiareolata, Aedes vittatus et Aedes mariae (populations de la côte tyrrhénienne). 


\section{Conclusions.}

Le milieu larvaire des moustiques peut être généralement approché assez bien au laboratoire, et les techniques d'élevage disponibles pour plusieurs espèces ont atteint un degré satisfaisant de standardisation et reproductibilité. Des progrès considérables restent toutefois encore à faire pour une meilleure connaissance des exigences alimentaires des larves, et du rôle joué dans leur nutrition par les micro-organismes associés aux aliments. L'importance de la photopériode devrait également être évaluée pour chaque espèce plus précisément qu'on ne l'a fait auparavant.

Les obstacles les plus fréquemment signalés dans la littérature concernant l'élevage des moustiques se manifestent au niveau des activités liées au cycle reproductif. De nombreuses espèces ne peuvent être élevées que dans la mesure où l'activité sexuelle, le repas de sang et la ponte sont stimulés ou forcés. A l'origine de ces anomalies de comportement, on trouve souvent des intéractions négatives entre milieu artificiel et rythmes endogènes. Nos connaissances dans ce domaine doivent progresser rapidement si l'on veut améliorer les techniques d'élevage.

Les perspectives d'élevage de masse sont limitées par l'eurygamie de plusieurs espèces. Dans ce cas, l'application de la technique de copulation forcée est nécessaire, et on ne voit pas actuellement comment cet obstacle pourrait être surmonté efficacement. De plus, le problème de la compétitivité des individus élevés au laboratoire, par rapport à ceux de la population naturelle semble être particulièrement important chez les moustiques. Le comportement sexuel et la mobilité sont fréquemment influencés par le milieu artificiel, et probablement modifiés par des processus sélectifs. L'étude génétique de ces phénomènes de comportement est à recommander.

\section{Bibliographie}

AKov (S.), 1962. - A qualitative and quantitative study of the nutritional requirements of Aedes aegypti larvae. J. Insect. Physiol., 8, 319-335.

Asahina (S.), 1964. - Food material and feeding procedures for mosquito larvae. Bull. Wld Hlth Org., 31, 465-466.

BAKER (R. H.), 1964. - Mating problems as related to the establishment and maintenance of laboratory colonies of mosquitos. Bull. Wld Hlth Org., 31, 467-468.

BATES (M.), 1941. - Laboratory observations on the sexual behavior of Anopheline mosquitoes. J. exp. Zool., 86, 153-173.

-, 1949. - The natural history of mosquitoes. MacMillan, New York, 379.

Baumhover (A. H.), Husman (C. N.) and Graham (A. J.), 1966. - Screw-worms. In: Smith C.N. Ed., Insect colonization and mass production. Academic Press, London, 533-554.

Clements (A.-N.), 1963. - The physiology of mosquitoes. Pergamon Press, London, 393 pp.

Coluzzı (M.), 1962. - Sulla modalità di applicazione della tecnica di McDaniel e Horsfall per l'accoppiamento artificiale delle zanzare. Riv. Malariol., 41, 75-78. 
-, 1964. - Maintenance of laboratory colonies of Anopheles mosquitos. Bull. Wld Hlth Org., 31, 441-443.

D'Alessandro (G.), Mariani (M.), Bruno-Smiraglia (C.), and Caravaglios (N.), 1961. Stenogamia dell'Anopheles labranchiae allevato in laboratorio. Riv. Malariol., 40, 267-274.

Dame (D. A.), Woodard (D. B.), Ford (H. R.), and WeidhaAs (D. E.), 1964. - Field behavior of sexually sterile Anopheles quadrimaculatus males. Mosquito News, 24, 6-14.

DanileVsKiI (A.S.), 1965. - Photoperiodism and seasonal development of insects. Oliver and Boyd, Edinburgh, $283 \mathrm{pp}$.

De Coursey (J. D.) and Webster (A. P.), 1952. - Effect of insecticides and other substances on oviposition by Aedes sollicitans. J. econ. Ent., 45, 1030-1034.

Gahan (J. B.), 1966. - Anopheles quadrimaculatus Say. In: Smith C.N. Ed., Insect colonization and mass production. Academic Press, London, 85-100.

- and Sмітн (C. N.), 1964. - Problems connected with raising mosquitos in the laboratory. Bull. Wld Hlth Org., 31, 445-448.

Gerold (J. L.), and LaArman (J. J.), 1967. - Behavioural responses to contact with DDT in Anopheles atroparvus. Nature, 215, 518-520.

Gregson (J. D.), 1966. - Ticks. In : Smith C.N. Ed., Insect colonization and mass production. Academic Press, London, 49-72.

Hazard (E. I.), Turner (R. B.) and Lofgren (C. S.), 1967. - Mosquito growth stimulating substances associated with infusions. J. med. Ent., 4, 455-460.

Knipling (E. F.), Laven (H.), Craig (G. B.), Pal (R.), Kitzmiller (J. B.), Smith (C. N.) and Brown (A.W. A.), 1968. - Genetic control of insects of public health importance Bull. Wld Hlth Org., 38, 421-438.

Kreutzer (R. D.), and KitZmiller (J. B.), 1969. - Colonization of Anopheles earlei Vargas. Mosquito News, 29, 589-590.

LAIRD (M.), 1956. - Studies of mosquitoss and fresh water ecology in the South Pacific. Roy. Soc. New Zeland Bull., 6, 213 pp.

Laurence (B. R.) and Suith (S. A.), 1958. - The breeding of Taeniorhynchus (subgenus Mansonioides) mosquitoes in the laboratory. Trans. roy. Soc. Trop. Med. Hyg., 52, 518-526.

Mattingly (P. F.), 1967. - Genetics of behaviour. In: Wright J. W. and Pal R., Ed., Genetics of insect vectors of disease. Elsevier, Amsterdam, 553-566.

McDaniel (I. N.), Horsfall (W. R.), 1957. - Induced copulation of Aedine mosquitoes. Sciences, 125, 745.

Morlan (H. B.), 1966. - Yellow fever mosquitoes. In : Smith C. N. Ed., Insect colonization and mass production. Academic Press, London, 585-599.

-, Mc Cray (E. M.), Kilpatrick (J. W.), 1962. - Field tests with sexually sterile males for control of Aedes aegypti. Mosquito News, 22, 295-300.

Muirhead-Thomson (R. C.), 1966. - Black flies. In: Smith C. N. Ed., Insect colonization and mass production. Academic Press, London, 127-144. 
Nielson (H. T.) and Nielson (E. T.), 1962. - Swarming of mosquitoes. Laboratory experiments under controlled conditions. Entomologia exp. appl., 5, 14-32.

Patterson (R. S.), Lofgren (C. S.), Boston (M. D.), $1968 a$. - The sterile male technique for control of mosquitos: a field cage study with Anopheles quadrimaculatus. Fla Ent., 51, 77-82.

_, _, —, 1968 b. - Sterile males for mosquito control : a field cage study with Culex pipiens quinquefasciatus. Mosquito News, 28, 540-544.

Roubaud (E.), 1932. - Recherches sur les variations trophiques et biologiques des peuplements de l'Anopheles maculipennis. Buli. Soc. Path. exot., 25, 755-762.

Trembley (H. L.), 1955). - Mosquito culture techniques and experimental procedures. Am. Mosq. Cont. Assoc. Bull., 3, 73 p.

Weidhaas (D. E.), Schmidt (C. H.) and Seabrook (E. L.), 1962. - Field studies on the release of sterile males for the control of Anopheles quadrimaculatus. Mosquito News, 22, 283-291. 